\title{
Renewal of NPCs requires MYC and $\beta$-catenin
}

$\beta$-Catenin signalling is required for the differentiation of multiple cell types in the developing kidney, but the mechanisms by which the pleiotropic functions of $\beta$-catenin are accomplished within different cell lineages remain unclear. Now, Thomas Carroll and colleagues show that $\beta$-catenin cooperates with the transcription factor MYC to direct nephron progenitor cell (NPC) fate towards renewal.

Previously, the team demonstrated that WNT9B- $\beta$-catenin signalling regulates the distinct transcriptional programmes of NPC renewal and differentiation in the developing kidney, but the mechanisms underlying these differential outcomes remained elusive. Hence, in their recent study they analysed transcription factor binding motifs that clustered near to each class of downstream targets and found enhancer elements for several of the genes expressed in the renewing NPCs. Using a combination of reporter cell lines and mouse models, they show that MYC activity is necessary to promote renewal but not differentiation of NPCs.

"In the kidney, there is probably no one gene that gets turned on in all the different cells in which $\beta$-catenin is active," says Carroll. "Each cell type will have a unique set of transcription factors that cooperate with $\beta$-catenin to turn on a unique set of targets." Next, the researchers want to identify other transcription factors, including those that direct NPCs towards differentiation, and elucidate how they modify $\beta$-catenin activity.

This study also has potential clinical applications. "Our work

suggests that $\beta$-catenin activity is needed to promote the renewal programme in kidney tissue engineering applications," explains Carroll. "Understanding of the mechanisms by which $\beta$-catenin promotes renewal instead of differentiation could be applied to in vitro and potentially in vivo approaches to generate unlimited numbers of NPCs." He also comments that $\beta$-catenin is frequently

In the

kidney, there is probably no one gene that gets turned on in all the different cells in which $\beta$-catenin is active overexpressed in Wilms tumour, the most common paediatric renal cancer, but the reason why active $\beta$-catenin promotes renewal rather than differentiation of NPCs in these tumours is unclear. Deciphering which $\beta$-catenin pathways are active in human Wilms tumours might lead to potential therapies.

Jack M. Heintze

ORIGINAL ARTICLE Pan, X. et al. Myc cooperates with $\beta$-catenin to drive gene expression in the nephron progenitor cells. Development http://dx.doi.org/10.1242/dev.153700 (2017) 